\title{
Introduction : la Franche-Comté ou la réalité d'un territoire en interface
}

\section{Alexandre Moine}

\section{(2) OpenEdition}

\section{Journals}

Édition électronique

URL : http://journals.openedition.org/rge/1431

DOI : $10.4000 / \mathrm{rge} .1431$

ISSN : 2108-6478

Éditeur

Association des géographes de l'Est

Édition imprimée

Date de publication : 1 juin 2007

ISSN : 0035-3213

\section{Référence électronique}

Alexandre Moine, «Introduction : la Franche-Comté ou la réalité d'un territoire en interface », Revue Géographique de l'Est [En ligne], vol. 47 / 3 | 2007, mis en ligne le 17 octobre 2011, consulté le 08 septembre 2020. URL : http://journals.openedition.org/rge/1431 ; DOI : https://doi.org/10.4000/rge. 1431

Ce document a été généré automatiquement le 8 septembre 2020.

Tous droits réservés 


\title{
Introduction : la Franche-Comté ou la réalité d'un territoire en interface
}

\author{
Alexandre Moine
}

1 Les territoires, systèmes complexes par excellence, posent la question de la compréhension des mécanismes sous-jacents aux organisations spatiales, et de l'échelle à partir de laquelle ils prennent sens. En abordant la Franche-Comté, comme une entité géographique régionale, force est de constater que cette question éminemment géographique, se pose à nous avec acuité. Au regard des dynamiques qui s'y révèlent, le cadre régional a bien un sens, mais il ne suffit pas à comprendre et à expliquer la totalité des mécanismes qui y sont à l'œuvre. Nous avons donc voulu au travers de ce numéro spécial consacré à la Franche-Comté, jeter un regard, non pas exhaustif, mais complet, sur quelques dynamiques régionales. Deux idées émergent lorsqu'on considère l'organisation spatiale régionale et les jeux d'acteurs sous-jacents :

- les dynamiques régionales s'expriment de plus en plus au travers des espaces urbains, qui s'organisent de manière plus ou moins homogène suivant les régions. À ce titre la FrancheComté pâtit d'une faiblesse de son armature urbaine, ce qui ne l'empêche néanmoins pas de développer un axe très structurant entre l'Alsace et Rhône-Alpes ;

- une région périphérique telle que la Franche-Comté, doit s'appuyer sur les régions limitrophes, et naturellement les opportunités que procurent certains aménagements contribuent à modifier en permanence l'organisation spatiale. Les acteurs en sont conscients, quels qu'ils soient et savent saisir les opportunités offertes, à l'inverse ils savent tout aussi bien éviter ou contourner les contraintes qui peuvent apparaître.

Pour comprendre comment ces processus sont à l'œuvre, nous avons choisi d'aborder le territoire régional sous l'angle des fonctions élémentaires que l'homme développe afin de satisfaire ses besoins élémentaires (habiter, approprier, exploiter et échanger) sachant que ceux-ci sont obligatoirement soumis à un pouvoir gestionnaire qui constitue la cinquième fonction. Les quatre premières contribuent à façonner l'organisation de l'espace par les objets qui en découlent (habitations, bâtiments, limites, activités diverses, réseaux), tandis que la cinquième révèle les systèmes organisationnels ou jeux d'acteurs locaux. Ces fonctions se combinent en permanence 
au gré des aménagements, des utilisations qui en sont faites et du pouvoir gestionnaire sous-jacent.

Abordons la question de l'habitat tout d'abord, au-delà des formes architecturales, ou sur un autre plan, de mécanismes qui sous-tendent les marchés du logement; intéressons nous au résultat en terme d'organisation spatiale, en abordant le phénomène d'étalement urbain. Il ne s'agit pas d'un processus isolé, mais bien d'un ensemble mécanismes complexes impliquant aussi bien des questions très sociales de modèle et de choix de vie, mais également des questions économiques liées au coût du foncier et à l'arbitrage rendu par le prix de l'énergie, ou encore de choix d'aménagement en répartissant les activités économiques aux périphéries des agglomérations. Le résultat est là, les tâches urbaines s'étalent et comme le soulignent les auteurs Jean-Philippe Antoni et Samy Youssoufi, « l'enchevêtrement des processus à l'œuvre conduit naturellement à une consommation toujours plus importante d'espaces par les aires urbaines. Il est intéressant dans le cas de la Franche-Comté de s'interroger sur les similitudes et les différences qui caractérisent les trois principales agglomérations, à savoir Besançon, Montbéliard et Belfort, dans un contexte de faible armature urbaine. Les résultats, au travers d'une analyse diachronique, montrent les différences de consommation de l'espace entre ces trois villes et on est surpris de constater que ce n'est pas là où la consommation et la population croissent le plus, que la consommation de l'espace est la plus importante. Encore une fois, des corrélations à priori évidentes sont démenties, il serait alors intéressant de comprendre pourquoi et comment les comportements des ménages sont influencés, qu'elle est la part des choix individuels, des orientations d'aménagement, ou du poids des traditions (l'usage de la voiture va de soi et modèle encore l'organisation de l'espace dans la cité du Lion).

4 L'appropriation de l'espace, deuxième fonction, nous conduit vers la question des mailles de l'aménagement et vers la gestion des territoires associés. Dans un cadre où l'émiettement communal maintes fois dénoncé, montre ses limites; il s'agit de s'interroger sur les moyens mis en œuvre à l'échelle régionale, afin de rationaliser les découpages et partant, les politiques locales d'équipement et d'aménagement. Les comportements départementaux mis en avant par Jean-Claude Chevailler et Philippe Signoret, montrent des différences, alors que la cohérence territoriale est aujourd'hui de rigueur. Il s'agit de créer le cadre aux rapprochements intercommunaux, euxmêmes sous-jacent à la mise en place de territoires de projets. Ceci suppose de s'entendre à différentes échelles, mais peut-on atteindre le consensus systématiquement, et peut-on pallier dans ce cadre, à la faiblesse de l'armature urbaine franc-comtoise ? Les auteurs nous montrent que si les systèmes de gouvernance mis en place dans le cadre intercommunal sont comparables, les régimes fiscaux font par contre la différence et on est moins prêt à partager dans certains départements que d'autres... Ceci met en avant la question de la faiblesse des ressources à des échelons où se gèrent maintenant de plus en plus d'équipements, et la nécessité de rationaliser les découpages intercommunaux, de gré ou de force. Finalement, la mise en place des intercommunalités révèle les tensions locales, et il apparaît clairement qu'il serait dommage que la faiblesse des moyens conduise à déclarer inefficace l'intercommunalité, au profit, comme le mentionnent les auteurs, d'une « recentralisation rampante». 
5 C'est dans ce cadre par ailleurs, que s'expriment aujourd'hui les activités, et deux articles nous éclairent sur deux aspects du développement économique régional, l'un en relation avec les microtechniques, et l'autre avec des activités sportives.

6 Ainsi, comment est-on passé d'une réalité industrielle horlogère à un développement territorial fondé sur la notion de microtechniques? Si la spécialisation microtechnique ne fait aucun doute pour les acteurs locaux qui utilisent abondamment cette image, la notion demeure aussi vague qu'ambiguë, mais suscite néanmoins une adhésion des collectivités locale et de l'État. Et comme le montre Sophie Carel, l'impulsion institutionnelle ne suffisant pas on constate parallèlement une organisation en termes de recherche et de transfert, et l'émergence de relations interentreprises. Ceci nous enseigne deux choses :

- les acteurs sont prépondérants, on ne décrète pas les coopérations, elles se nouent lentement et se nourrissent d'une infinité de relations formelles et informelles ;

- un système productif localisé est fondé sur des relations de proximité, son identification n'est pas évidente s'il s'appuie sur une grande variété de produits et sur des acteurs disséminés en divers territoires.

7 De la réalité à une image pour forger une réalité, la boucle de rétroaction n'est pas facile à enclencher, mais il semble que les microtechniques commencent à devenir un objet intéressant de développement territorial en Franche-Comté.

Une autre activité retient notre attention, elle est tertiaire, liée aux loisirs et souvent négligée en temps qu'outil de développement local. Le sport passionne, mais au-delà de l'engouement pour tel ou tel championnat, ou manifestation d'envergure, on oublie souvent le rôle structurant des activités sportives. Il est clair que le milieu est prégnant, laissant émerger des pratiques spécifiques, portées de manière emblématique à la fois par le département du Jura et la région Franche-Comté, comme le ski nordique. Mais au-delà, la Franche-Comté est une zone tampon entre les territoires du sport traditionnel et les territoires méridionaux, où le sport spectacle pâti naturellement de la faiblesse de l'armature urbaine déjà maintes fois relevée. Loïc Ravenel s'interroge ensuite sur les spécificités sportives régionales, autour de trois disciplines (judo, karaté et football), interrogées à différentes échelles, qui révèlent que plaine et/ou montagne, ruralité et/ou urbanité sont des clés de lecture non exclusives, qui doivent être complétées par des apports sociologiques et historiques afin d'expliquer des comportements locaux qui surprennent quelques fois. Ceci montre une fois de plus que les territoires doivent être abordés comme des systèmes complexes et que les perspectives de développement sont assujetties à de nombreuses tendances bien difficiles à anticiper conjointement. Dernière fonction et pas des moindres celle des échanges, évoquée ici sous l'angle des nouveaux aménagements ferrés à grande vitesse. Si la Franche-Comté n'a jamais été un carrefour ferroviaire, elle n'a jamais été non plus une périphérie oubliée. L'organisation de l'armature urbaine actuelle, son importance, s'expliquent en partie par le développement du réseau ferré. Il apparaît clairement aujourd'hui que la nouvelle LGV Rhin-Rhône ne permettra pas d'améliorer totalement l'accessibilité de la Haute-Saône, qui peut s'appuyer sur les aménagements récents liés au TGV Est situé plus au nord. Au travers d'une analyse fouillée Jean-Louis Mignot nous montre par ailleurs le rôle que pourrait jouer la Haute-Saône, afin de lever des verrous nord-sud extérieurs à la région, en délestant le trafic des voies ferrées de l'Est tout en désenclavant le département. Encore une fois ici, il apparaît clairement que les échelles de l'aménagement sont plurielles et qu'il s'agit d'envisager plus globalement certains 
engagements. Dans le cadre franc-comtois, ceci met également en évidence le rôle charnière de cette région entre les influences rhénanes, lorraines, bourguignonnes et rhône-alpine, renforçant l'idée qu'il est plus pertinent de se placer en appui concerté sur ses périphéries, plutôt que de privilégier un aménagement dans le seul cadre des limites régionales.

9 Ces différents articles illustrent la complexité sous-tendante l'aménagement du territoire, qui aujourd'hui doit de surcroît offrir la garantie d'une cohérence dans les démarches. L'organisation de l'espace géographique est une chose, les jeux d'acteurs en regard, une autre ; mais les deux sont indissociables, liés par la boucle de rétroaction de l'aménagement. On pressent bien à la lecture de ces différents articles, qu'en arrière de ce qu'il nous est donné d'observer, de multiples interrelations se nouent entre des décideurs, des utilisateurs, qui suivant le recul qu'ils ont des phénomènes auront plus ou moins de capacité à apprécier, à s'approprier, les opportunités qui s'offrent à eux. Ce regard pertinent que posent les géographes, clair, sans compromis, faisant émerger de multiples questionnements, traduit des méthodes performantes, des outils efficaces, mais surtout une manière de représenter la réalité complexe qui nous entoure afin de mieux la maîtriser.

\section{AUTEUR}

\section{ALEXANDRE MOINE}

Professeur à l'Université de Franche-Comté - Laboratoire ThéMA 